\title{
Classification of Resting, Anticipation and Movement States in Self-Initiated Arm Movements for EEG Brain Computer Interfaces
}

\author{
Miguel Rodrigo, Luis Montesano, Javier Minguez
}

\begin{abstract}
In the last years, there has been an increasing interest in using Brain Computer Interfaces (BCI) within motor rehabilitation therapies that use robotic devices or functional electro stimulation to help or guide the efforts of the patient to move her body. A crucial step of these therapies is to provide help to the user just when she is actually trying to accomplish a certain motion or task One of the most promising applications of BCI systems in this context is its ability to measure the user intentions and actions to trigger the rehabilitation devices accordingly. This paper studies the single-trial classification based on EEG measurements of three basic states during the execution of self-initiated motion: rest, motion preparation (or anticipation) and motion. We conducted an experiment where the participants had to reach at their will eight different locations from a fixed starting position. Results for seven healthy subjects show that it is possible to achieve good classification rates given that features are carefully selected for each subject and for each pair of states.
\end{abstract}

\section{INTRODUCTION}

One of the application contexts of brain-computer interfaces is in robotic related rehabilitation programs, where they could provide a medium to decode the human intentions to modulate the robot intervention. For instance, this is the case in motor rehabilitation programs in stroke patients where it has been suggested that the brain-computer interface could play a key role by providing human cognitive aspects such as motor intention, perception of the feedback, and higher level motivational states as the attention [1]. These are important factors with a key role in any seamless integration between the human and the rehabilitation robot with potential to favor a natural compliance and adherence of the patient in the therapy process.

Many motor therapies are based on the development of repetitive analytical movements of the targeted limb. Within this movement process there are at least three macro-states of interest: the rest state, the preparation of motion, and the motion execution. The on-line monitoring with a braincomputer interface of these states could provide a valuable information to design any robot-mediated therapy for control (triggering of events), for monitoring of the process, and as a measure of compliance and engagement in fully automated therapies. The decoding of these three macro-states simultaneously from EEG has not been addressed yet and it is the objective of this paper. An important observation

Luis Montesano and Javier Minguez are with the Instituto de Investigación en Ingeniería de Aragón (I3A) and Dpto. de Informática e Ingeniería de Sistemas (DIIS), Universidad de Zaragoza, Spain. E-mail: montesano,jminguez@unizar.es. M. Rodrigo is with Bit\&Brain Technologies, Zaragoza, Spain. E-mail: mrodrigo@bitbrain.es. This work has been partially supported by projects HYPER-CSD2009-00067 and DPI200914732-C02-01 funded by the Spanish Government is that although the rest and the movement states could be decoded from other sensory modalities such as the EMG, the preparation of motion is the only state with anticipatory information of the intention of the subject and can only be decoded from brain activity. In addition to this, the on-line decoding of this state is gaining attention in brain-computer interfaces since it is also present during motor imagery [2] (which is one of the key mental tasks for operation).

Although there is not research in decoding the three states simultaneously, there is significant research in differentiating between the rest state from the movement (and imagination of the movement), and in between the rest state and the motor preparation. It is well known that during the movement execution there is an event-related de-synchronization in motor areas $(12-15 \mathrm{~Hz})$ [3] and also there is slow cortical activity involved (motor-related potentials) [4]. Usually the sensorymotor synchronization/desynchronization is the key feature used to differentiate between the rest and movement states in real or motor imagery [5]. In addition to this, several studies have demonstrated the appearance of EEG activity preceding human voluntary movement. These signals are associated to motor task preparation and are dissimilar from those during the actual execution. Similar to the movement, there is the event-related (de)synchronization (ERD/ERS) and a slow activity denoted the readiness potential. Several studies have focused in distinguishing between the anticipation of movement of limbs [6], [7], [8], [9] in time or frequency domains. An important point of these last works that report decoding of anticipatory motor rhythms is that they are not self-paced and the subject is always cued. The contribution of the present paper is a simultaneous decoding of the three states in a self-paced context (i.e. the subjects decides when to start the motion and choose among different types of motion), which is the ecological working setting.

\section{Methodology}

\section{A. Experiment}

Seven right-handed male students of the University participated voluntary in the experiments (age range 25-32 years) after the protocol was approved by the Institutional Review Board of the University of Zaragoza. Subjects were seated in a comfortable chair in front of the reaching apparatus (Figure 1a). They were instructed to move the right arm from the homing location to a self-chosen location among eight possible locations and then come back to the homing position. This process was self-paced approximately every 7.5 secs on average (minimum 2.8 and maximum 9.7 secs). During the reaching operation, subjects were asked to maintain a natural, 


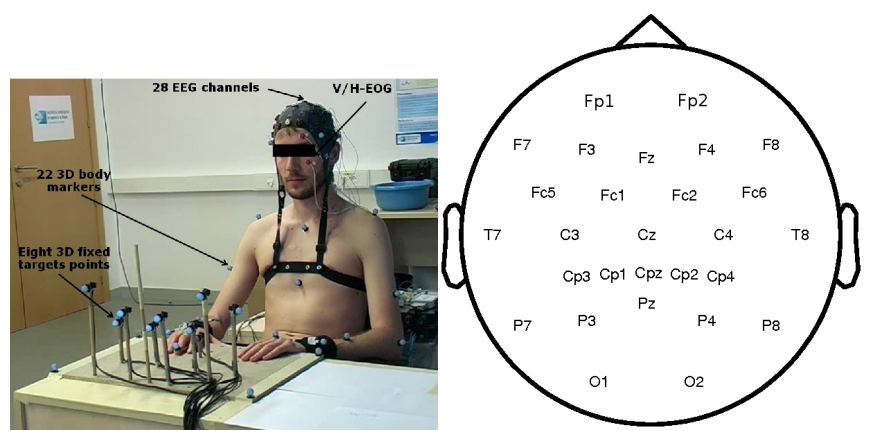

(a)

(b)

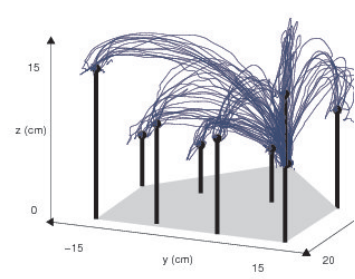

(c)

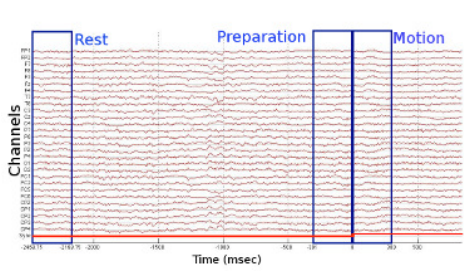

(d)
Fig. 1. (a) Experimental setup. The user sits in front of the reaching apparatus with a fixed start position and set of eight predefined positions. (b) Sensor placement. (c) Trajectories executed by the users. (d) Acquired signals including synchronization signal obtained from the buttons at the positions. The blue rectangles illustrate the extracted examples of each class (rest, preparation, motion) for each trial.

relaxed and constant posture; and to minimize blinking while maintaining the gaze fixed in the appropriate point of the apparatus. In the remaining time they were allowed to blink and relax. The first condition was executed in five blocks of 5 minutes each, where the subjects executed around 130 reaching operations to the eight locations (minimum after removing non valid examples 75 and maximum 196). Figure 1(c) shows the trajectories executed by subject 1 .

\section{B. Data recording}

EEG activity was recorded with a gTec system (2 gUSBamp amplifiers synchronized) with 28 electrodes distributed according to the 10/10 international system (Figure 1b), with the ground on FPz and the reference on the left earlobe. Vertical and horizontal EOG were also recorded. The EEG and EOG signals were digitalized with a sampling frequency of $256 \mathrm{~Hz}$, power-line notch-filtered and lowpass-filtered at $60 \mathrm{~Hz}$.

In addition to EEG measurements, the system also recorded time markers corresponding to the beginning and end of the arm reaching motion. This information was obtained using buttons both at the home and the target positions that were synchronized with the EEG measurements. Based on the motion markers, each trial was segmented in three different parts corresponding to rest, preparation and motion conditions with a fixed duration of 0.3 seconds each (see Figure 1(d)). Thus, each trial provides one example of each class.

\section{Feature extraction and classification}

Let $X \in \mathcal{R}^{C \times T}$ correspond to the acquired EEG signal of a class example where $C$ is the number of channels and $T$ the number of samples. One of the key aspects to distinguish rest, preparation and motion conditions is to select features from the EEG measurements that have the relevant information to distinguish between the classes. According to previous studies, this information is the event-related desynchronization/synchronization (ERD/ERS) in motor areas $(12-15 \mathrm{~Hz})$ [3]. There is also important activity in lower frequencies corresponding to slow cortical potentials involved in the motor preparation (motor-related potentials) [4]. In principle, this type of information should be discriminative enough to distinguish between the three classes of interest (rest, preparation and motion). Firstly, as mentioned in the introduction ERD/ERS has been used to distinguish between rest and either motion or preparation. Secondly, the slow potentials are different for the preparation (or anticipation) of the motion and the motion execution.

In addition to the raw data $X$, the feature selection process applied two different filters to the raw data. This pre-processing step is common to improve the classification performance. The filters were:

- Re-referencing filter: The EEG measurements are rereferenced to one specific channel. By repeating this process for all the channels, we obtain a total of $C$ filters that generate signals $X_{B i} \in \mathcal{R}^{C \times T}$, with $i \in 1$..C.

- Laplacian filter: The EEG signal $X$ was filtered using a $3 \times 3$ discrete Laplace operator to obtain $X_{L} \in \mathcal{R}^{C \times T}$.

Once $X, X_{B i}, X_{L}$, The selection of the best filter for each subject was based on the $r^{2}$ test of the spectral density between pairs of classes (i.e. rest vs. preparation, rest vs. motion and preparation vs. motion). For each trial and channel, we estimated the spectral density of the different filtered signals using the periodogram. Then, for each pair de classes we selected just one filtered signal among $X, X_{B i}, X_{L}$ based on the $r^{2}$ maximum value of channel C3 (which is one of the most representative electrodes related to motion of the right arm). The final selection was done by visual inspection based on the periodograms (see Section III). Based on $r^{2}$ values, we also selected the range of frequencies $f_{r}$ of the periodogram with the highest value for the filtered channel $\mathrm{C} 3$. In addition to this channel, all the channels of the selected filter that were above a certain threshold were also included in the feature vector.

The last step before training the classifier consisted in processing the selected channels in the time domain. First, a Butterworth band-pass filter was applied in the selected range $f_{r}$. This filtered temporal signal was rectified and smoothed (which empirically has shown to be a good way to obtain signals, and then characteristics, to increase the statistical difference between conditions). As in the frequency domain, an $r^{2}$ test was used to select the best signal among the four different types resulting from applying sequentially to the filtered signal, the band-pass filter, the rectification and the smoothing. 

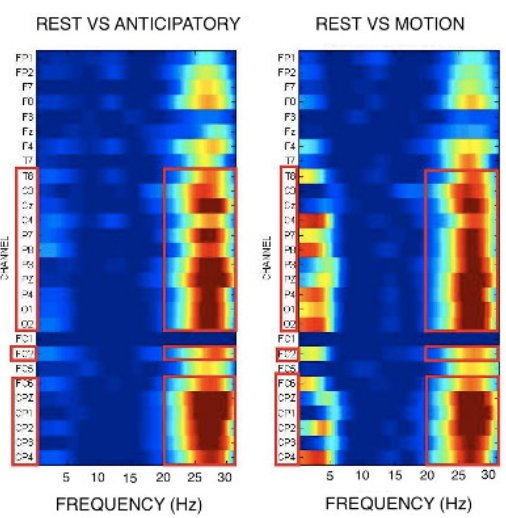

(a)

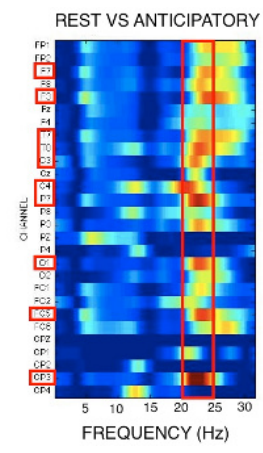

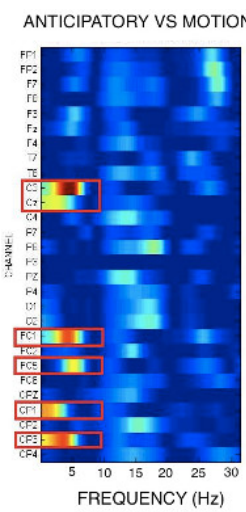

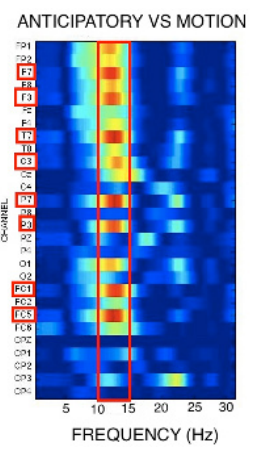

(c)
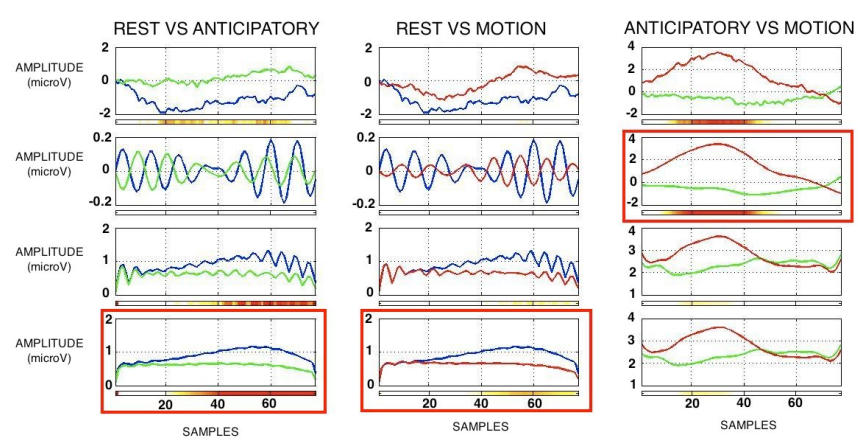

(b)

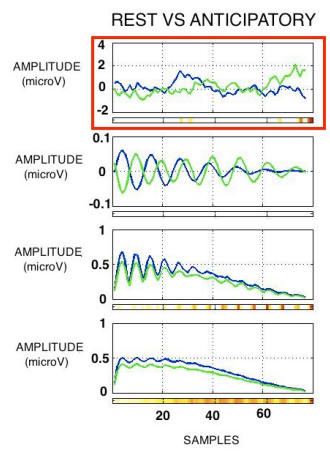

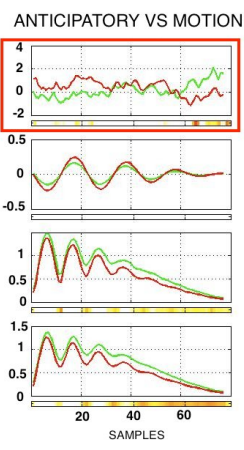

(d)

Fig. 2. Selected features. (a) $r^{2}$-test values of the periodogram of the selected filters for each pair of classes (rest vs. motion, rest vs. preparation, preparation vs. motion) for subject one. The red boxes indicate the range of frequencies and channels used for classification, (b) Signals in the temporal domain for channel C3 of the filter selected for each pair of classes. Signals are averaged over all the examples of each class. From top to bottom, $i$ ) selected features, $(i i)$ filtered in the selected frequency band, $(i i i)$ rectified and $(i v)$ smoothed. The corresponding $r^{2}$-test is shown for each case. The red box indicates the final features fed to the classifier. (c) $r^{2}$-test values of the periodogram for subject 4 . (d) Final features fed to the classifier for subject 4 .

The final selected features, subsampled to $64 \mathrm{~Hz}$, were used to train a (multi-class) LDA classifier. Since the feature vector dimension can be larger or close to the number of examples of each class, we used a shrinkage estimator for the covariance matrix [10]. When classifying between two classes, the feature vector was formed by the temporal signal of that specific pair (e.g. rest and motion). For the simultaneous classification of the three conditions, the feature vector was the concatenation of the filters selected for each pair of classes.

\section{RESULTS}

\section{A. Selected Features}

We first analysed the selected features for each user. Recall that filters were selected independently for each subject and for each pair of conditions. Table III-A shows the selected filters, where $B_{i}$ indicates re-referenced with respect to channel $i$. The first remark is that in all cases (subjects and pairs of classes), re-referenced filters were the ones with best $r^{2}$ compared to raw measurements $X$ and to Laplacian filtered channels $X_{L}$. Second, for every pair of classes and every subject there were multiple filtered channels that contained discriminative information. The table shows that the best selected filters vary significantly among subjects as well as the corresponding frequency ranges.
Figures 2(a) and 2(c) show the $r^{2}$-test values of the periodogram for the selected filters for the subjects one and four who had the largest and smallest statistical differences, respectively. Recall that they correspond to different filters as indicated in Table III-A. For the best subject, subject one, the information selected to separate rest and preparation corresponds to an ERD de-synchronization in high beta bands (around $25 \mathrm{~Hz}$ ) at the central and parietal zones. In the case of rest versus motion, the figure shows a similar pattern to the previous one plus some relevant statistical difference in low frequencies $[0-5] \mathrm{Hz}$. The latter is probably due to slow potentials related to motion as described in [11]. Finally, the $r^{2}$ values between preparation and motion show a completely different structure where the most useful information appears in low frequencies around $5 \mathrm{~Hz}$. As in the previous case, these differences are probably caused by slow potentials such as the lateralized readiness potentials. Figure 2(b) show the averaged signal features in the time domain for channel C3.

The results for subject four shows that his best selected features have quite a different structure and indicated that the different conditions are not as different as those for subject one (see Fig. 2(c)). Furthermore, the channels and frequency bands are not as consistent with the literature as the ones from other subjects such as subject one. Consequently, the temporal signals shown in Fig. 2(d) are also less discriminative (as we will corroborate when analyzing the classification 
TABLE I

FILTERS AND FREQUENCY RANGE SELECTED FOR EACH SUBJECT AND PAIR OF CLASSES

\begin{tabular}{|c|c|c|c|}
\hline Subj. & Rest VS Antici. & Rest VS Motion & Antici. VS Motion \\
\hline 1 & $\mathrm{~B}_{F C 1},[20-30] \mathrm{Hz}$ & $\mathrm{B}_{F C 1},[20-30] \mathrm{Hz}$ & $\mathrm{B}_{P 3},[0-10] \mathrm{Hz}$ \\
\hline 2 & $\mathrm{~B}_{F Z},[25-30] \mathrm{Hz}$ & $\mathrm{B}_{F C 2},[10-20] \mathrm{Hz}$ & $\mathrm{B}_{F C 2},[10-20] \mathrm{Hz}$ \\
\hline 3 & $\mathrm{~B}_{C P 3},[20-30] \mathrm{Hz}$ & $\mathrm{B}_{F 7},[0-5] \mathrm{Hz}$ & $\mathrm{B}_{C P 3},[20-30] \mathrm{Hz}$ \\
\hline 4 & $\mathrm{~B}_{C P z},[20-25] \mathrm{Hz}$ & $\mathrm{B}_{C P 1},[10-15] \mathrm{Hz}$ & $\mathrm{B}_{C P z},[10-15] \mathrm{Hz}$ \\
\hline 5 & $\mathrm{~B}_{P 4},[10-15] \mathrm{Hz}$ & $\mathrm{B}_{O 1},[15-25] \mathrm{Hz}$ & $\mathrm{B}_{O 1},[17-20] \mathrm{Hz}$ \\
\hline 6 & $\mathrm{~B}_{P 8},[5-10] \mathrm{Hz}$ & $\mathrm{B}_{F P 1},[0-10] \mathrm{Hz}$ & $\mathrm{B}_{F P 1},[0-10] \mathrm{Hz}$ \\
\hline
\end{tabular}

TABLE II

CLASSIFICATION RESULTS FOR EACH PAIR OF CONDITIONS PER SUBJECT

\begin{tabular}{|c|c|c|c|}
\hline Subject & Rest VS Antici. & Rest VS Motion & Antici. VS Motion \\
\hline 1 & 69.59 & 67.86 & 80.78 \\
\hline 2 & 67.30 & 81.01 & 75.01 \\
\hline 3 & 72.01 & 72.57 & 68.47 \\
\hline 4 & 58.63 & 55.39 & 63.18 \\
\hline 5 & 50.88 & 48.70 & 58.36 \\
\hline 6 & 66.93 & 56.54 & 61.15 \\
\hline Mean & 64.22 & 63.68 & 67.70 \\
\hline
\end{tabular}

results).

\section{B. Classification results}

Here we present the classification results and discuss two different cases: $(i)$ the classification of pairs of classes independently; and $(i i)$ the joint classification of the three classes.

Table III-B summarizes the classification results of each pair of classes. The mean accuracy for each pair is around $64 \%$. There is very little variation between the different pairs of classes. However, subjects behave differently with an average of $74 \%$ and $72 \%$ for the two best subjects (subject 2 and subject 1) and an average of $52 \%$ and $59 \%$ for the two worst cases (subject 5 and 4).

We now analyze the results of the LDA classifier in the multi-class case. Table III-B shows the confusion matrix averaged for the six subjects. The classification rates for each class are similar to the ones obtained in the previous case with a degradation of almost $10 \%$ on average from $65 \%$ in the two class case to $55 \%$ in the three class one. In the latter, the subject one is the best with a mean recognition rate of $72 \%(68 \%, 69 \%, 78 \%$ for rest, preparation and motion, respectively). The worse subject is subject 4 with a mean recognition rate of $40 \%(32 \%, 41 \%, 47 \%$ for each class). These results are as expected since the analysis of the features already revealed that there were important differences between the subjects in terms of statistical difference among the classes of the best features. Indeed, a deeper analysis of the EEG measurements of subjects 4 and 5 suggest that

TABLE III

MEAN CONFUSION MATRIX AVERAGED FOR ALL SUBJECTS

\begin{tabular}{|c|c|c|c|}
\hline & Rest & Anticipation & Motion \\
\hline Rest & 54.9937 & 23.5145 & 21.4918 \\
\hline Anticipation & 23.6410 & 54.7408 & 21.6182 \\
\hline Motion & 22.6296 & 20.4804 & 56.8900 \\
\hline
\end{tabular}

the ERD/ERS could not be clearly identified and there was no statistical difference in the frequency bands of the slow potentials in any of the filters. It is also worth to mention that those subjects that had the best classification rates (e.g subjects 1 and 2) kept their performance in the three classes case, while those with the lower rates (e.g. subjects 4 and 5) degraded their performance considerably.

\section{CONClusions}

This paper addresses the classification of the basic phases (rest, preparation and motion) involved in self-paced motion using EEG measurements obtained through a BCI system. The method explicitly builds and selects features for each pair of classes according to their statistical difference based on the $r^{2}$-test. The results show that it is possible to achieve reasonable classification rates around $70 \%$ by appropriately selecting the features for each of the six subjects.

Planned future work explores two different directions. First, we are currently working on more automatic feature selection processes that does not require any visual inspection by the therapist. Second, although motion is self-initiated by the user, classification still requires synchronized markers to segment the signal. We plan to investigate structured prediction techniques to label the full sequence as recorded from the BCI system.

\section{REFERENCES}

[1] K. P. Tee, C. Guan, K. Keng Ang, K. Soon Phua, C. Wang, and H. Zhang;, "Augmenting cognitive processes in robot-assisted motor rehabilitation," in Proceedings of the 2nd Biennial IEEE/RAS-EMBS International Conference on Biomedical Robotics and Biomechatronics, Scottsdale, USA, 2008.

[2] Christa Neuper, Reinhold Scherer, Miriam Reiner, and Gert Pfurtscheller, "Imagery of motor actions: Differential effects of kinesthetic and visual-motor mode of imagery in single-trial eeg," Cognitive Brain Research, vol. 25, no. 3, pp. 668 - 677, 2005.

[3] G. Pfurtscheller and F. Lopez da Silva (Eds.), "Event-related synchronization," in Handbook of Electroencephalography and Clinical Neurophysiology, 1999.

[4] M. Kutas and E. Donchin, "Preparation to respond as manifested by movement-related brain potentials," in Brain Research, 1980, number 95.

[5] C. Neuper and G. Pfurtscheller, "Event-related dynamics of cortical rhytms: frequency-specific features and functional correlates," in International Journal of Psychophysiology, 2001, pp. 41-58.

[6] Valerie Morash, Ou Bai, Stephen Furlani, Peter Lin, and Mark Hallett, "Classifying eeg signals preceding right hand, left hand, tongue, and right foot movements and motor imageries," Clinical Neurophysiology, vol. 119, no. 11, pp. $2570-2578,2008$.

[7] B.O. Peters, G. Pfurtscheller, and G. Edlinger, "Automatic differentiation of multichannel eeg signals," IEEE Transactions on Biomedical Engineering, vol. 48, no. 1, 2001.

[8] B. Blankertz, G. Dornhege, C. Schafer, R. Krepki, J. Kohlmorgen, K.R. Muller, F. Losch V. Kunzmann, and G. Curio, "Boosting bit rates and error detection for the classification of fast-paced motor commands based on single-trial eeg analysis," IEEE Transactions Neural Systems Rehabilitation Engineering, vol. 11, no. 127, 2003.

[9] M. Krauledat, G. Dornhege, B. Blankertz, F. Losch, G. Curio, and K.R. Mller, "Improving speed and accuracy of brain-computer interfaces using readiness potential features," Engineering in Medicine and Biology Society, pp. 4511-4515, 2004.

[10] J.B. Copas, "Regression, prediction and shrinkage," Journal of the Royal Statistical Society. Series B (Methodological), vol. 45, no. 3, pp. 311-354, 1983.

[11] S. Waldert, H. Preissl, E. Demandt, C. Braun, N. Birbaumer, A. Aertsen, and C. Mehring, "Hand movement direction decoded from meg and eeg," J. Neuroscience, vol. 28, no. 4, pp. 1000-1008, 2008. 